\title{
Ruptured and Unruptured Intracranial Aneurysms - Surgical Outcome
}

\author{
Gary A. Dix, William Gordon, Anthony M. Kaufmann, Ian S. Sutherland and \\ Garnette R. Sutherland
}

\begin{abstract}
Background: The treatment of unruptured, intracranial aneurysms has been the topic of debate. Although recent studies have advocated surgical intervention for unruptured aneurysms, the risk of such treatment in comparison to outcome from ruptured aneurysms has not been established. Method: This retrospective study examines the outcome of 134 patients with 179 ruptured and unruptured intracranial, saccular aneurysms treated by a single surgeon. Results: Of the 98 ruptured aneurysms where early surgical intervention was undertaken (less than 48 hours post hemorrhage), 70 had an excellent outcome, 13 were good, four were moderate, two poor and nine patients died postoperatively. Outcome assessment in these cases was correlated to preoperative neurological status. Patients who presented with unruptured aneurysms fell into two categories: symptomatic and asymptomatic. Seven incidental, asymptomatic aneurysms were clipped concurrently to the surgical isolation of the culprit lesion following subarachnoid hemorrhage without influencing outcome, whilst, for varying reasons, eight unruptured aneurysms were not operated upon. Of the remaining 66 surgically treated, unruptured aneurysms, 64 had an excellent postoperative result, one was good (persisting right incomplete third nerve palsy) and one was moderate (left hemiparesis). Thirteen of these aneurysms were symptomatic, whilst 21 were asymptomatic, multiple aneurysms requiring secondary elective repair and 32 were true incidental aneurysms. Conclusion: Unruptured aneurysms less than $25 \mathrm{~mm}$ in size may be safely, surgically treated relative to the expected natural history and, certainly, with less risk than operative intervention upon ruptured cerebral aneurysms.
\end{abstract}

RÉSUMÉ: Résultats de la chirurgie des anévrismes intracrâniens rupturés et non rupturés. Introduction: Le traitement des anévrismes intracrâniens est un sujet de controverse. Bien que des études récentes favorisent le traitement chirurgical des anévrismes non rupturés, le risque associé à ce traitement comparé à celui d'un anévrisme rupturé n'a pas été établi. Mêthodes: Il s'agit d'une étude rétrospective examinant le résultat du traitement de 179 anévrismes sacciformes intracrâniens, rupturés et non rupturés, chez 134 patients traités par le même chirurgien. Résultats: Des 98 anévrismes rupturés traités précocement par chirurgie (dans les 48 heures de l'hémorragie), le résultat a été excellent pour 70 anévrismes, bon pour 13, moyen pour 4, mauvais pour 2 et 9 . patients sont décédés dans les suites postopératoires. L'évaluation du résultat chez ces cas était corrélée à l'état neurologique préopératoire. Nous avons divisé les patients qui se présentaient avec un anévrisme non rupturé en deux catégories, selon qu'ils étaient symptomatiques ou non. Sept anévrismes asymptomatiques découverts fortuitement ont été clippés au moment de la chirurgie pour une autre lésion ayant provoqué une hémorragie sous-arachnoidienne, sans influencer l'issue de la chirurgie. Huit anévrismes non rupturés n'ont pas été traités chirurgicalement pour différentes raisons. Des 66 autres anévrismes non rupturés traités chirurgicalement, les résultats postopératoires ont été excellents chez 64 , bons chez un (paralysie résiduelle incomplète du troisième nerf crânien) et moyen chez un (hémiparésie gauche). Trente-quatre étaient des anévrismes multiples exigeant une chirurgie élective, dont treize étaient symptomatiques et 21 étaient asymptomatiques. La découverte des 32 autres avait été fortuite. Conclusions: L'issue de la chirurgie des anévrismes non rupturés de moins de $25 \mathrm{~mm}$ se compare favorablement à celle de leur histoire naturelle et présente certainement moins de risques que la chirurgie des anévrismes cérébraux rupturés.

Can. J. Neurol. Sci. 1995; 22: 187-191

The management of unruptured, intracranial aneurysms remains a contentious neurosurgical issue. This is fostered, in part, by the limited available data regarding the natural evolution of berry aneurysms, ${ }^{1-3}$ the optimal size at which aneurysmal surgery should be undertaken ${ }^{4,5}$ and the long term outcomes of patients who have had operative treatment for unruptured aneurysms. ${ }^{6}$ Nonetheless, the surgical management of aneurysms prior to rupture represents the greatest potential for reducing the
From the Department of Clinical Neurosciences (Neurosurgery), The University of Calgary. Calgary (G.A.D., G.R.S.); Department of Radiology (Neuroradiology). The University of Manitoba, Winnipeg (W.G., I.S.S.); Department of Neurological Surgery, University of Pittsburgh, Pittsburgh, Pennsylvania (A.M.K.) RECEIVED DECEMBER 12, 1994. ACCEPTED ON FEBRUARY 23, 1995.

Reprint requests to: Dr. Garnette Sutherland, Department of Clinical Neurosciences, Foothills Hospital, 1403 - 29 Street N.W., Calgary, Alberta. Canada T2N 2T9 
incidence of subarachnoid hemorrhage (SAH) and its invariably catastrophic sequelae. ${ }^{57.8}$ Contemporary advances in diagnostic imaging modalities and microsurgical techniques have resulted in increased detection of unruptured aneurysms and decreased morbidity and mortality associated with operative repair. Despite the obvious advantages of early intervention, it remains prudent to review the risks of such surgery and compare them to both the risks of spontaneous bleeding in patients harboring untreated aneurysms, as well as the consequences of surgery on patients in whom rupture has already occurred. $5.7,9-11$ Increasingly, current studies are advocating early, elective intervention for unruptured aneurysms in patients with low risk for complications. ${ }^{12.13}$

To further establish the importance of surgically treating unruptured, intracranial aneurysms, this study compares the surgical outcome in patients presenting to a single surgeon with either ruptured or unruptured aneurysms.

\section{Case Material}

Between 1984 and 1994, 134 patients were treated by the senior author (GRS) for ruptured and unruptured aneurysms at The University of Manitoba, Winnipeg, Manitoba and The University of Calgary, Calgary, Alberta. These patients represented a total of 179 aneurysms, of which there were 98 ruptured and 81 unruptured aneurysms. The ages of the patients in this series ranged from 20 to 77 years (mean 48.5; S.D. \pm 13.0 ) with a female to male ratio of $2: 1$. This study includes only those patients in whom early surgical intervention (less than 48 hours post $\mathrm{SAH}$ ) was performed by a single surgeon to reduce the tendency towards individual selection bias, and selection criteria were based on the modified Botterell scale and the general medical condition of the patient prior to operation. Only aneurysms less than $25 \mathrm{~mm}$ were considered for this series. Aneurysms of less than $3 \mathrm{~mm}$ diameter were not included in this study in view of their presently uncertain, but presumably low, potential for spontaneous rupture.

\section{RESULTS}

Ninety-eight patients presented with SAH and were subsequently diagnosed with cerebral angiography as having ruptured a berry aneurysm. The ages of these patients ranged from 20 to 70 years (mean 46.3; S.D. \pm 12.5 ) with female to male ratio of 2:1. Aneurysm location was as follows: anterior cerebral artery, 33; middle cerebral artery, 24; posterior cerebral artery, five; internal carotid, 22; and vertebro-basilar circulation, 14 (Table 1). Aneurysmal size was taken to be the maximal, saccular diameter and was measured with scaled angiography; 25 were classified as small (less than $7 \mathrm{~mm}$ diameter); 59 were medium ( 8 to $14 \mathrm{~mm}$ diameter); and 14 were large (15 to $25 \mathrm{~mm}$ diameter) (Table 2). Following surgery, patients were assessed at between 12 to 16 weeks and categorized according to their outcome (Figure 1). An excellent result was accorded to 70 patients; whilst 13 cases were good; four moderate; and two were given a poor outcome. In nine cases, the patients died following surgery (Table 3 ).

In this series, 81 unruptured aneurysms were discovered incidentally in 41 patients ( 22 of whom presented with multiple aneurysms). The mean age for this group was 54.6 years with S.D. \pm 13.5 and ages ranged from 24 to 77 , whilst the female to
Table 1:

\section{ANEURYSM LOCATION}

\begin{tabular}{lrrrr}
\hline Location & \multicolumn{2}{c}{ Ruptured } & \multicolumn{2}{c}{ Unruptured } \\
\hline ACA & 33 & $(34)$ & 18 & $(22)$ \\
MCA & 24 & $(25)$ & 31 & $(38)$ \\
PCA & 5 & $(5)$ & 15 & $(19)$ \\
IC & 22 & $(22)$ & 9 & $(11)$ \\
V-B & 14 & $(14)$ & 8 & $(10)$ \\
\hline Total & 98 & $(100)$ & 81 & $(100)$ \\
\hline
\end{tabular}

Table 2:

ANEURYSMAL SIZE

\begin{tabular}{lcccc}
\hline Size & Ruptured & Unruptured \\
\hline Small $(3$ to $7 \mathrm{~mm})$ & 25 & $(26)$ & 35 & $(43)$ \\
Medium $(8$ to $14 \mathrm{~mm})$ & 59 & $(60)$ & 31 & $(38)$ \\
Large $(15$ to $25 \mathrm{~mm})$ & 14 & $(14)$ & 15 & $(19)$ \\
\hline Total & 98 & $(100)$ & 81 & $(100)$ \\
\hline
\end{tabular}

\section{Outcome Scale}

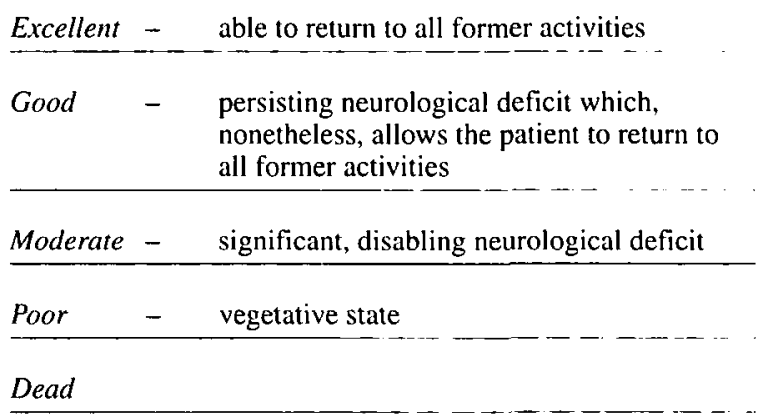

Figure 1.

male ratio was 2.6:1. Of these aneurysms, 31 were discovered in the vicinity of the middle cerebral artery; 18 on the anterior cerebral artery; 15 on the posterior cerebral artery; nine on the internal carotid artery; and eight in the vertebro-basilar territory (Table 1). In determining the sizes of the aneurysms, 35 were classified as small; 31 were medium; and 15 large (Table 2).

The unruptured aneurysms were subdivided into groups depending upon their presentation and management. In eight cases, no surgery was performed with reasons ranging from death of the patient between emergency surgery for aneurysmal rupture and proposed elective surgery for incidentally discovered, additional, unruptured aneurysms, 2 ; inoperable, intracavernous aneurysms, 2; aneurysms less than $3 \mathrm{~mm}$ and considered too small for surgery, 3; and one case in which the patient refused further treatment. In seven patients, because of intraoperative accessibility, the incidentally discovered aneurysms were clipped concurrently with the surgical isolation of the ruptured aneurysm and the long term outcome for these patients was deemed to be dependent upon their recovery from the SAH. The 
Table 3: Ruptured aneurysms - outcome vs. clinical grade

Ruptured Aneurysms ( $=98$ )

Clinical Guide

$\begin{array}{lcrrrrr}\text { Outcome } & \text { \# Pts. } & \text { I } & \text { II } & \text { III } & \text { IV } & \text { V } \\ \text { Excellent } & 70 & 33 & 27 & 10 & - & - \\ \text { Good } & 13 & 3 & 4 & 6 & - & - \\ \text { Moderate } & 4 & 1 & 1 & 2 & - & - \\ \text { Poor } & 2 & - & - & - & 1 & 1 \\ \text { Dead } & \mathbf{9} & - & \mathbf{2} & \mathbf{1} & \mathbf{4} & \mathbf{2}\end{array}$

remaining 66 aneurysms were classified as either symptomatic or asymptomatic, i.e., aneurysms discovered as a result of mass effect or those patients in whom diagnosis of the berry aneurysms was a truly fortuitous, incidental occurrence.

Thirteen patients presented with symptomatic intracranial aneurysms detected by cerebral angiography (Table 4). The sizes of these aneurysms ranged from 9 to $25 \mathrm{~mm}$ and accounted for a variety of presenting signs and symptoms, including cranial nerve compression, hemicranial headaches and ataxia. In 11 cases, the aneurysm was successfully ablated by application of a surgical clip, whilst in two patients, aneurysmal trapping was effected by extracranial to intracranial (EC/IC) arterial bypass. All 13 patients demonstrated an excellent postoperative result with resolution of their preoperative signs and symptoms.

In 14 patients, 21 unruptured aneurysms discovered during angiography for a previous $\mathrm{SAH}$, but not surgically accessible during the first operation, were electively operated upon at a later time. Thirteen patients had excellent results and one was assessed as being moderate. In the latter case, a 46-year-old female, who had previously undergone successful surgery on a left-sided $12.5 \mathrm{~mm}$ aneurysm following $\mathrm{SAH}$, presented for elective clipping of a second right-sided $10 \mathrm{~mm}$ aneurysm. Intraoperatively, the neck of the aneurysm was noted to be calcified and required crushing before successful application of the clip could be carried out. Following surgery, the patient presented with a left hemiparesis which improved slowly but still necessitated the use of a cane for assisted ambulation.

The remaining 32 aneurysms were classified as true incidental aneurysms, discovered in patients for reasons other than mass effect or previous $\mathrm{SAH}$. These included lesions suspicious for aneurysm on CT brain imaging during routine work up for other neurological problems (e.g., dementing illness, Parkinson's disease, persisting headaches and progressive, unexplained hemiparesis). Angiography during investigation of carotid stenosis, transient ischemic attacks and severe epistaxis also harvested a number of incidental aneurysms. Sizes ranged from $51020 \mathrm{~mm}$ and, in seven patients, multiple aneurysms were present. The aneurysms were treated by surgically clipping the aneurysmal neck and outcome was classified as excellent for 31 aneurysms and good in one patient, who presented with an incomplete third cranial nerve palsy following surgical isolation of a $20 \mathrm{~mm}$ posterior communicating artery aneurysm.

Of the 98 patients surgically treated for ruptured intracranial aneurysms, $72 \%$ had an excellent outcome; $13 \%$ had minor complications; $6 \%$ suffered major morbidity; and $9 \%$
Table 4: Unruptured aneurysms - outcome vs. presentation

Unruptured Aneurysms $(n=66)$

\begin{tabular}{llll} 
Outcome & $\begin{array}{l}\text { Secondary } \\
\text { Repair } \\
(\mathbf{n = 2 1})\end{array}$ & $\begin{array}{l}\text { Symptomatic } \\
(\mathbf{n = 1 3})\end{array}$ & $\begin{array}{l}\text { Incidental } \\
(\mathbf{n}=\mathbf{3 2})\end{array}$ \\
Excellent & 20 & 13 & 31 \\
Good & - & - & 12 \\
Moderate & 11 & - & - \\
Poor & - & - & - \\
Dead & - & - & - \\
\hline $110 \mathrm{~mm}$ calcified MCA aneurysm & & \\
$220 \mathrm{~mm}$ PoCoA aneurysm &
\end{tabular}

died. Outcome was noted to be inversely correlated to clinical grade at the time of presentation (Table 3 ). By comparison, patients who underwent elective, surgical management of routinely discovered, unruptured berry aneurysms had a $97 \%$ chance of an excellent outcome. A minor complication was evident in one patient (1.5\%) postoperatively, and one patient suffered major morbidity (Table 4). Despite the relatively small number of patients involved in this study, no significant difference in outcome following surgical management of symptomatic and asymptomatic unruptured aneurysms was seen. In the group of patients with good or excellent results following surgical repair of ruptured aneurysms, $29 \%$ had lesions less than $7 \mathrm{~mm}$ in size; 55\% had aneurysms 8 to 14 $\mathrm{mm}$; and $16 \%$ were greater than $15 \mathrm{~mm}$. Among the 15 patients with major morbidity or death, one aneurysm was less than $7 \mathrm{~mm}(7 \%)$; 11 were 8 to $14 \mathrm{~mm}(73 \%)$; and three greater than $15 \mathrm{~mm}(20 \%)$. Of the unruptured aneurysm patients who had a good or excellent outcome following elective surgery, $39 \%$ were less than $7 \mathrm{~mm}$; $44 \%$ were 8 to $14 \mathrm{~mm}$ in diameter; and $17 \%$ greater than $15 \mathrm{~mm}$. The single patient from the incidental aneurysm category with major postoperative morbidity had a $10 \mathrm{~mm}$ aneurysm.

\section{Discussion}

\section{Limitations of Data}

In this study, a relatively small number of patients was reviewed and intergroup comparisons should, therefore, be interpreted with caution. In addition, a single surgeon's operative results are not necessarily applicable to the neurosurgical community. Nonetheless, we believe that the conclusions from this study are valid and help to address the controversy surrounding the management of patients with unruptured, intracranial aneurysms.

During the past 45 years, there had been no appreciable decline in the incidence of SAH or in the morbidity of the disease, despite a statistical reduction in the annual morbidity and mortality rates associated with surgical treatment of ruptured intracranial aneurysms during the same period. ${ }^{14-16}$ This latter development may be attributed to several factors including improved diagnostic modalities allowing for earlier surgical intervention, better pre and postoperative medical management of the complications associated with SAH, refinement of surgical 
techniques, and better judgment with respect to optimal timing for aneurysmal surgery. ${ }^{5,6.14}$ With an annual estimated incidence of 10.9 cases per 100,000 population, ${ }^{13,17}$ a mortality rate of $60 \%$ for untreated, ruptured aneurysms ${ }^{15}$ and 2 to $30 \%$ for aneurysms in which surgical intervention was carried out (dependent upon preoperative grading and time from $\mathrm{SAH}$, or last rebleed, to surgical management), it is apparent that a more effective approach to this condition needs to be developed and pursued. ${ }^{6,18}$

An increasing amount of attention has been focused on the management of unruptured, incidental aneurysms. By ablating the aneurysms prior to spontaneous rupture, the devastating effects of SAH would be avoided. Surgical or endovascular intervention is not without its own inherent risks, however, and these need to be weighed against the complications associated with potential, future aneurysmal rupture. In their review of 50 patients with multiple aneurysms in whom a previous ruptured aneurysm had been treated, Winn et al. found the risk of rupture of an intact aneurysm to be approximately $1 \%$ per annum. ${ }^{13}$ In a study of 142 patients in whom multiple or single unruptured aneurysms were incidentally discovered without prior $\mathrm{SAH}$, Juvela et al. documented an estimated $1.4 \%$ annual rupture rate. They also noted that, whilst there was a correlation between size (maximal diameter) of the largest aneurysm and risk of future rupture in patients with multiple aneurysms, there was no critical diameter above which the propensity for rupture increased..$^{19}$

Wiebers and coworkers reported on a long term followup study of 161 unruptured aneurysms and found that the probability for rupture in aneurysms less than $10 \mathrm{~mm}$ was extremely low, and suggested that no surgical intervention be considered in such cases. ${ }^{12.20}$ Crompton, in reviewing the brains of 289 patients dying from berry aneurysmal rupture, determined that the critical size at which an aneurysm was likely to become unstable and rupture was $4 \mathrm{~mm}^{21}$ Wiebers felt that the observation of Crompton could be attributed to the natural propensity of the aneurysm to partially collapse at the time of rupture, and because of subsequent thrombus formation in the aneurysmal sac. ${ }^{12}$ Several papers have subsequently disputed the recommendations of Wiebers and his colleagues, reporting on a number of cases of previously intact, angiographically-demonstrated aneurysms of less than $10 \mathrm{~mm}$ which have ruptured spontaneously. ${ }^{3.22-24}$ In an angiographical study of the growth of intracranial aneurysms, Allcock and Canham found that there was no consistent rate of growth of aneurysmal sacs.' The conclusion to be drawn from these findings is that small, asymptomatic aneurysms do possess an inherent risk of rupture and should be considered for definitive, surgical management where possible.

In a report published in 1970 by Heiskanen and Martila, the researchers concluded that the risks of surgical intervention in unruptured aneurysms outweighed the natural risks of SAH. ${ }^{25}$ However, several subsequent studies have advocated early, elective intervention for symptomatic and incidentally-discovered, asymptomatic unruptured aneurysms less than $25 \mathrm{~mm}$ in diameter. ${ }^{2.79-11,22.26}$ In the series of Solomon et al., size, and not location, was the main variable predicting the incidence of complications during surgery. However, this in itself should not be a major contraindication for surgical intervention. ${ }^{7}$ In the hands of a skilled surgeon, intervention on aneurysums of 10 to
$25 \mathrm{~mm}$ carries a major morbidity risk of less than $5 \%$, whilst those less than $10 \mathrm{~mm}$ have an equivalent risk of $1 \%$." Age of the patient, co-morbid medical conditions and informed consent are further considerations to be taken under advisement by the surgeon when selecting patients for such surgery.

The results of this study support the consensus of opinion of contemporary neurosurgeons. Major morbidity and mortality was significantly less in patients who underwent elective, surgical repair of unruptured aneurysms compared to those in whom aneurysmal rupture was followed by emergency surgical treatment. Based on an annual rupture rate of $1-1.4 \%$ for intact aneurysms, a significant benefit following surgical intervention would be realized in two to three years, providing a major surgical morbidity of less than or equal to $2 \%$ was attained. Among the ruptured aneurysms, $25 \%$ were only 3 to $7 \mathrm{~mm}$ in size, emphasizing the importance of considering surgery in unruptured aneurysms of similar size.

\section{ConCLusion}

Providing there are no medical or surgical contraindications, unruptured, intracranial berry aneurysm less than $25 \mathrm{~mm}$ in size should be isolated from the cerebral circulation. The benefits of controlled, surgical ablation of these lesions outweigh the risks of such intervention, as well as the morbidity and mortality which can be anticipated following SAH when an expectant approach is adopted.

\section{REFERENCES}

1. Allcock JM, Canham PB. Angiographic study of the growth of the intracranial aneurysms. J Neurosurg 1976; 45: 617-621.

2. Deruty R, Pelisson-Guyotat I, et al. Surgical management of unruptured, intracranial aneurysms - personal experience with 37 cases. Acta Neurochir 1992; 119: 35-41.

3. Kassell NF, Torner JC. Size of intracranial aneurysms. Neurosurgery 1983; 12: 29I-297.

4. Kassell NF, Drake CG. Timing of aneurysmal surgery. Neurosurgery 1982; 10: 514-519.

5. Kassell NF, Torner JC, et al. The international cooperative study on the timing of aneurysmal surgery. Part $I$ and 2. J Neurosurg 1990; 73: 18-47.

6. Sundt Jr TM, Shigeaki K, et al. Results and complications of surgical management of 809 intracranial aneurysms in 722 cases. J Neurosurg 1982; 56: 753-765.

7. Solomon RA, Fink ME, et al. Surgical management of unruptured intracranial aneurysms. J Neurosurg 1994; 80: 440-446.

8. Winn HR, Richardson AE, et al. The long term prognosis in untreated cerebral aneurysms: Il. Late morbidity and mortality. Ann Neurol 1978; 4: 418-426.

9. Moyes PD. Surgical treatment of multiple aneurysms and of incidentally-discovered, unruptured aneurysms. J Neurosurg 1971; 35: $291-295$.

10. Salazar JL. Surgical treatment of asymptomatic and incidental intracranial aneurysms. J Neurosurg 1980; 53: 20-21.

11. Samson DS, Hodosh RM, et al. Surgical management of unruptured asymptomatic aneurysms. J Neurosurg 1977; 46: 731-734.

12. Wiebers DO, Whisnant JP, et al. The significance of unruptured intracranial saccular aneurysms. J Neurosurg 1987; 66: 23-29.

13. Winn HR, Almaani WS, et al. The long term outcome in patients with multiple aneurysms. Incidence of late hemorrhage and implications for treatment of incidental aneurysms. J Neurosurg 1983; 59: 642-651

14. Ingall TJ, Whisnant JP, et al. Has there been a delcine in subarachnoid hemorrhage mortality? Stroke $1989 ; 20: 718-724$.

15. Phillips LH, Whisnant JP, et al. The unchanging pattern of subarachnoid hemorrhage in a community. Neurology 1980;30: 1034-1040. 
16. Sundt Jr TM, Whisnant JP. Subarachnoid hemorrhage from intracranial aneurysms. N Engl J Med 1978; 299: 116-122.

17. Pakarinen S. Incidence, etiology and prognosis of primary subarachnoid hemorrhage: a study of 589 cases diagnosed in a defined urban population during a defined period. Acta Neurol Scand 1967; (Suppl.) 29: 1-128.

18. Weir B, Petruk K, et al. Management mortality related to the timing of surgery for anterior circulation aneurysms. $\ln$ : Auer L.M., ed. Timing of Aneurysm Surgery. Berlin: Walter de Gruyter, 1985: 165-175.

19. Juvela S, Porras M, et al. Natural history of unruptured, intracranial aneurysms: a long term followup study. J Neurosurg 1993; 79: 174-182.

20. Wiebers DO, Whisnant JP, et al. the natural history of unruptured intracranial aneurysms. N Engl J Med 1981; 304: 696-698.
21. Crompton MR. Mechanism of growth and rupture in cerebral berry aneurysms. Br Med J 1966; 1: J138-1142.

22. Roger LA. Intracranial aneurysm size and potential for rupture correspondence. J Neurosurg 1987; 67: 475-476.

23. Rosenorn J, Eskesen V. Does a safe size-limit exist for ruptured intracranial aneurysms? Acta Neurochir 1993; 121:113-118.

24. Schievink WI, Piepgras DG, et al. Rupture of previously documented small asymptomatic saccular intracranial aneurysms - report of three cases. J Neurosurg 1992; 76: 1019-1024.

25. Heiskanen O, Marttila I, et al. Risk of rupture of a second aneurysm in patients with multiple aneurysms. J Neurosurg 1970; 32: 295-300.

26. Nakagawa T, Hashi $K$. The incidence and treatment of asymptomatic, unruptured cerebral aneurysms. J Neurosurg 1994; 80: 440-446. 